\title{
La cornemuse du Trás-os-Montes (Portugal)
}

\section{Anne Caufriez}

\section{OpenEdition \\ Journals}

Édition électronique

URL : http://journals.openedition.org/ethnomusicologie/2339

ISSN : 2235-7688

Éditeur

ADEM - Ateliers d'ethnomusicologie

Édition imprimée

Date de publication : 1 janvier 1989

Pagination : 165-182

ISBN : 2-8257-0178-5

ISSN : 1662-372X

Référence électronique

Anne Caufriez, «La cornemuse du Trás-os-Montes (Portugal) », Cahiers d'ethnomusicologie [En ligne],

2 | 1989, mis en ligne le 15 septembre 2011, consulté le 05 mai 2019. URL : http://

journals.openedition.org/ethnomusicologie/2339 


\title{
LA CORNEMUSE \\ DU TRÁS-OS-MONTES (PORTUGAL)
}

\author{
Anne Caufriez
}

Lorsqu'on emprunte cette route sinueuse de campagne qui mène de Miranda do Douro à Bragance, la première présence humaine s'offrant au regard est celle de ces pâtres qui s'appuient avec indolence sur leur grand bâton. Figure centrale de ces hauts plateaux qui forment la frange nord du Trás-os-Montes (province nord-est du Portugal), le pâtre appartient à toutes les saisons. En été, il garde les chèvres, les moutons ou les bœufs, abrité par la seule constellation des étoiles. En hiver, il transhume vers les bergeries pour veiller le gros bétail convoité par les loups. Au Trás-os-Montes, il n'y pas de cornemuseur qui ne soit berger. Ainsi, plusieurs de ces musiciens m'ont-ils conté l'extrême dureté de ces veillées d'hiver au cours desquelles ils se retrouvent au bord d'un brasero, frissonnant dans leur couverture au milieu des glaces et des neiges. En hiver comme en été, le berger vit isolé, à l'écart du village qui lui a confié la garde du troupeau collectif. Dans ce contexte, la cornemuse est un passe-temps précieux et une source inestimable de divertissement.

La cornemuse de cette province est essentiellement un instrument des hauts plateaux, à savoir les plateaux mirandais et les plateaux situés au nord et au nord-ouest de Bragance. Toute cette barrière montagneuse représente une région où se sont cristallisées certaines des plus vieilles coutumes du Portugal. Nous nous trouvons au cœur d'une société agro-pastorale perpétuant un système économique de type communautaire qui reposait jusqu'à très récemment sur le troc.

Le berger représente un pôle essentiel de cette société. Son métier s'inscrit dans une chaîne de relais désignée par l'assemblée du village qui lui confie la garde du troupeau commun. Si d'antan chaque village pouvait se prévaloir d'un cornemuseur de renom figurant de manière privilégiée dans la généalogie d'une famille, à l'heure actuelle ce musicien est en voie de disparition. Mais si, évoluant vers le salariat, la fonction de berger se raréfie elle aussi, les bergers restent beaucoup plus nombreux que les cornemuseurs.

A combien d'expériences désastreuses n'avons-nous pas été confrontés lors de nos missions dans cette région, en 1978 et 1983 ? Beaucoup de cornemuseurs 


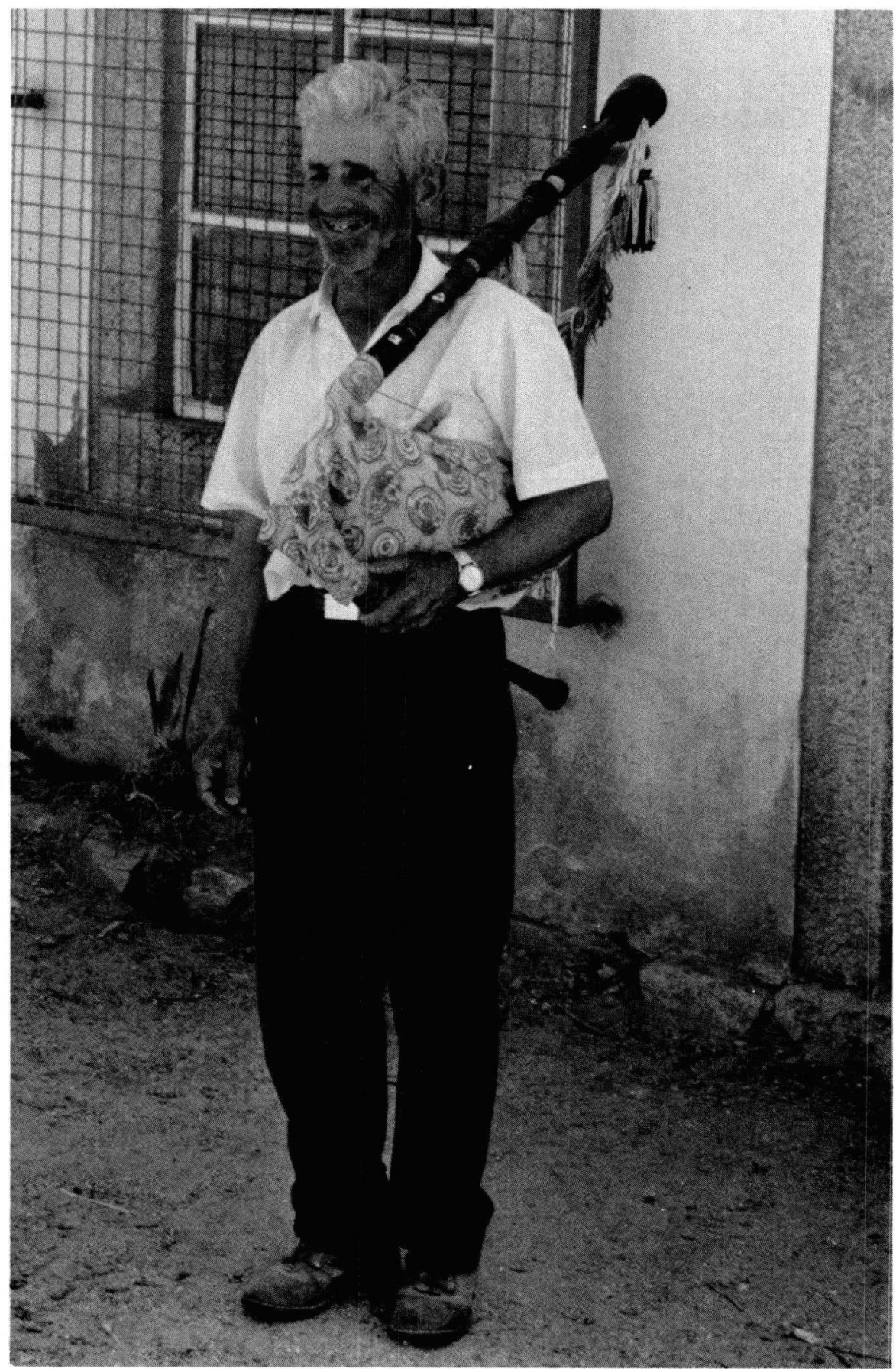

Fig. 1: P. Pereira João (66 ans), cornemuseur du village de Póvoa. Photo: A. Caufriez, 1978. 


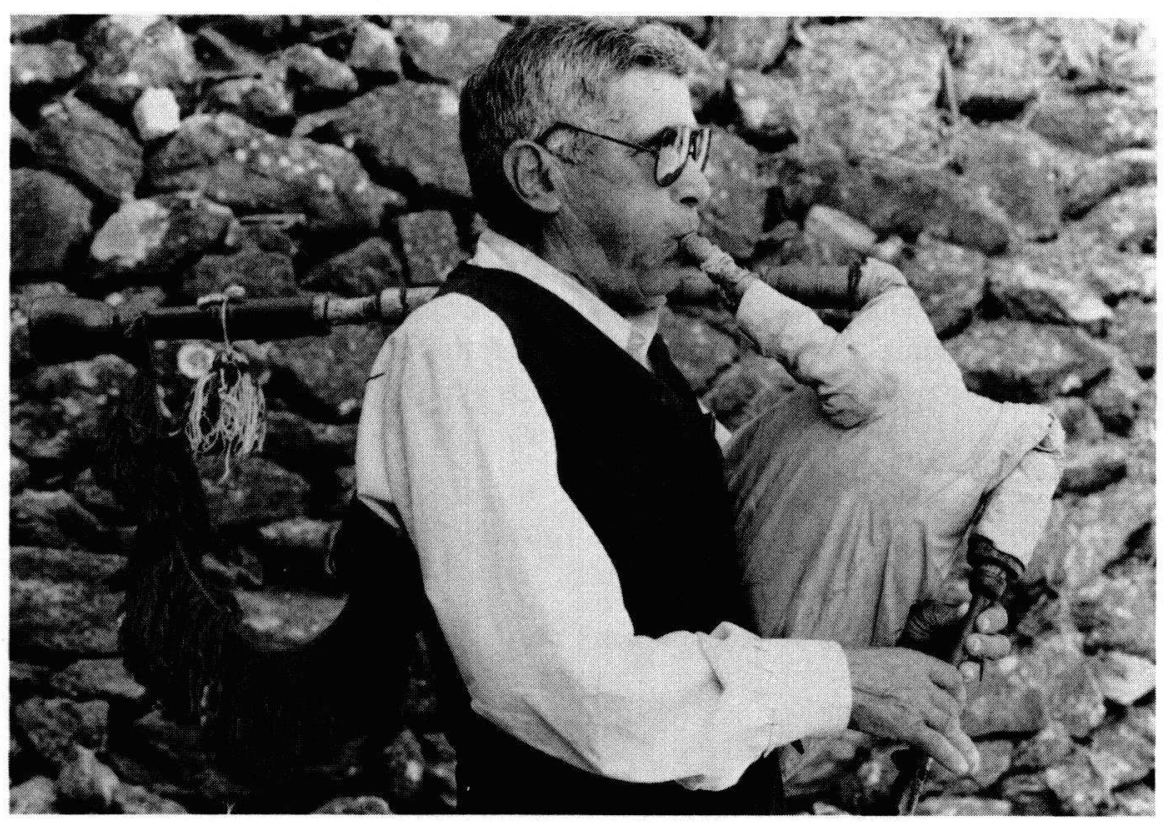

Fig. 2: A. Gil do Cubo (65 ans), du village de São Julião. Photo: A. Caufriez, 1983.

connus étaient morts ou privés de souffle parce que malades; d'autres n'avaient plus que des instruments décomposés; enfin, les derniers étaient des musiciens manquant d'entraînement, voire de talent. Sur une vaste zone géographique, nous n'en avons sélectionné que six qui soient de valeur appéciable. Au village, l'heure n'est plus aux sortilèges de la cornemuse, dont l'apprentissage est réservé aux seuls bergers et suit la voie patrilinéaire.

Seul le curé du village de Duas Igrejas, le Padre Mourinho, constitue une exception. Il, anime son propre groupe de musiciens et de danseurs dont il colporte les talents sur la scène de divers festivals. Ainsi, le vieux cornemuseur de ce village a-t-il troqué son art ancestral contre le formalisme d'un groupe aux séductions pittoresques. La sévère noblesse des vieilles danses galiciennes qui évoluaient aux stridences d'une cornemuse imparfaite mais rustique, s'égarant parfois dans des formules inattendues, a disparu à tout jamais.

Le moment semble donc venu d'opérer une synthèse sur la vie de cette cornemuse transmontaine, la plus ancienne et la plus archaïque du Portugal. Quelles sont ses caractéristiques et ses fonctions dans la vie paysanne passée et présente? Quel est l'état de la tradition entre 1978 et 1983 ? Nous n'avons, en effet, plus eu l'occasion de retourner dans cette province depuis lors.

\section{Les sources historiques}

Les cordophones sont absents de l'instrumentarium traditionnel du Trásos-Montes. Le principal instrument mélodique, la cornemuse, sert et exprime 
toute la musique et la culture de cette province. Elle est désignée sous le nom de Gaita de Foles ${ }^{1}$

La cornemuse portugaise représente, pour certains organologues, une lointaine réminiscence de la culture celtique qui marqua en particulier la Galice médiévale. On se rappellera que celle-ci est le berceau du premier royaume portugais né au XII ${ }^{e}$ siècle. En effet, si l'on se réfère aux témoignages historiques parvenus jusqu'à nous, la cornemuse du Trás-os-Montes et du Minho semble bien issue de ce vieux fonds galaïco-portugais. Ce sont essentiellement les sources iconographiques (sculptures, bas-reliefs, enluminures) qui viennent éclairer la chronologie de l'instrument dans cette aire culturelle, dont la Galice espagnole nous livre les indices les plus anciens.

- La cornemuse est déjà représentée sur un chapiteau galicien du $\mathrm{XI}^{\mathrm{e}}$ siècle, celui de Mellid (La Coruña), où l'on observe un instrument étroitement apparenté à celui du Trás-os-Montes actuel.

- Sur les enluminures du Recueil des Cantigas de Santa Maria (Galice, XIII ${ }^{\mathbf{e}}$ siècle), jongleurs et ménestrels s'adonnent au jeu d'une cornemuse semblable à la précédente.

- Plus tard, au XIVe siècle, l'instrument est aussi représenté dans les mains d'un berger, celui de l'autel en bas-relief offert au Roi Dom João I de Castille (Musée de Guimaraẽs, Minho).

- $\mathrm{Au} \mathrm{XVe} \mathrm{siècle,} \mathrm{on} \mathrm{observe} \mathrm{encore} \mathrm{la} \mathrm{figure} \mathrm{du} \mathrm{cornemuseur} \mathrm{sur} \mathrm{divers} \mathrm{reta-}$ bles de la Nativité, dont celui de la Grâce à Torres Vedras (Minho).

Que ce soit dans les scènes de la Nativité de la fin du Moyen Age ou dans des représentations ultérieures, la cornemuse est à chaque fois l'attribut du berger, ce qui se vérifie encore de nos jours (Oliveira 1966: 169-70).

\section{La morphologie}

Dans la palette des cornemuses que présente l'Europe, Anthony Baines distingue la cornemuse atlantique (ou celtique) par son large chalumeau de forme conique et par la présence de tambours d'accompagnement. L'accompagnement de cette partie rythmique (un ou deux tambours) est en effet attesté aussi bien en Galice espagnole et au nord du Portugal, qu'en Ecosse ou en Bretagne. Par ailleurs, les cornemuses espagnoles de Galice et des Asturies et les cornemuses portugaises du Minho et du Trás-os-Montes sont étroitement apparentées (Baines 1960: 103, 111).

Gaita signifie «chalumeau», et foles, «soufflet», «instrument qui sert à souffler» ou «sac de cuir"m. 
La cornemuse portugaise est à bourdon unique, présentant une même structure fondamentale. Seuls quelques détails de ses éléments constitutifs diffèrent d'une région à l'autre. Quant à l'instrument du Trás-os-Montes, il correspond au type le plus simple de la cornemuse galicienne: la grileira. C'est la cornemuse la plus petite et la plus aiguë de la Galice espagnole, nous dit Riego (la tonique du chalumeau donne ré). La grileira coexiste en Galice avec deux autres types, la redonda (tonique: la) et la tumbal (tonique: si bémol) de dimension plus grande (Riego 1960: 6).

Traditionnellement, la cornemuse transmontaine présente un réservoir d'air, fole, constitué d'une peau de chèvre, de chevreau ou de mouton ${ }^{2}$. La partie antérieure de la peau, correspondant au cou de l'animal et à ses pattes avant, est utilisée pour fixer le chalumeau, le tuyau d'insufflation et le bourdon, alors que la partie postérieure est refermée par un lien (on ligature l'extrémité de la peau à l'aide de fil) (Baines 1960: 108).

Sur le plan décoratif, la cornemuse des bergers transmontains a toujours sa peau recouverte d'un tissu aux motifs variés, vestimenta, alors que le tuyau du bourdon est orné d'une frange colorée, franjas. Ce tissu évite en outre de salir les vêtements du musicien. Si c'est la vieille cornemuse que nous avons eu le privilège d'enregistrer, il faut préciser qu'à l'heure actuelle, plusieurs instruments du Trás-os-Montes proviennent des manufactures de Galice espagnole. Tuyaux de bois tournés à la machine et réservoirs en caoutchouc constitués d'une chambre à air de pneu de voiture sont les signes d'une nouvelle facture populaire. Le réservoir recouvert d'une housse rouge aux franges jaunes et bleues évoque les trois couleurs du drapeau galicien.

Quels sont les éléments constitutifs de la cornemuse transmontaine traditionnelle? Elle se compose de trois tuyaux en bois d'olivier ou de buis:

- Le tuyau d'insufflation (assoprete), constitué de deux parties: à l'extrémité supérieure, l'embouchure (boquilha), et à l'extrémité inférieure, le tenon (cano) taillé dans la même pièce de bois, qui s'insère dans la souche (bocal) du réservoir d'air. Ce tenon se termine par un clapet de cuir fin et flexible (bucha), destiné à faire entrer l'air insufflé dans le réservoir, sans toutefois le laisser ressortir.

- Le chalumeau (ponteiro): il est de forme conique, à l'intérieur comme à l'extérieur, alors que son pavillon présente le profil d'une cloche. L'extrémité supérieure de ce chalumeau s'insère dans la souche bocal du réservoir d'air par l'intermédiaire du tenon (cano), taillé dans la même pièce de bois. Ce tenon est muni d'une anche double (palheta). Celle-ci est constituée de deux languettes de roseau en forme d'ongle, taillées en pointe dans la partie inférieure pour entrer dans un petit tuyau métallique auquel elle est ligaturée. Ce tuyau s'insère dans le tenon du chalumeau. Le chalumeau comporte

2 D'après le cornemuseur Gil Do Cubo, la peau de chèvre est bien supérieure à celle du mouton. Les cornemuses que nous avons enregistrées possèdaient un récipient à air dont le sac est en peau de chèvre. 


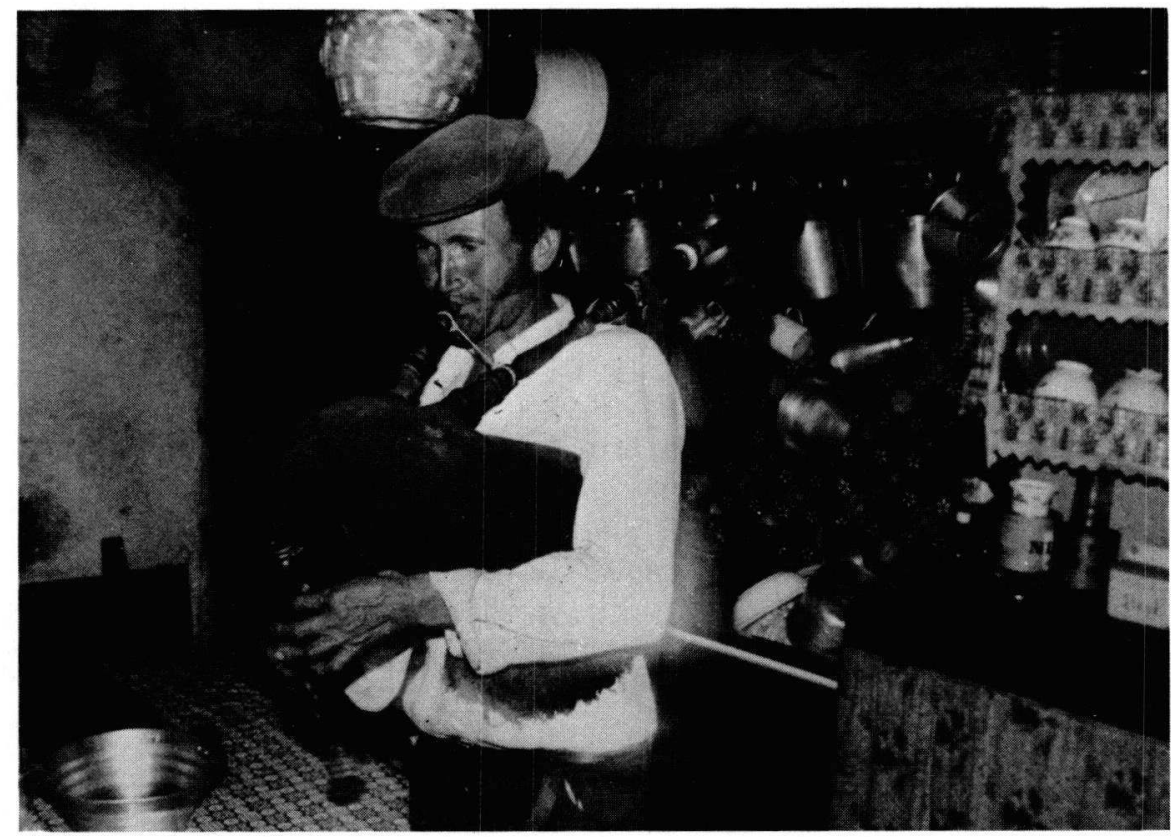

Fig. 3: E. F. Veiga (42 ans), cornemuseur du village de Deilão. Photo: A. Caufriez, 1983.

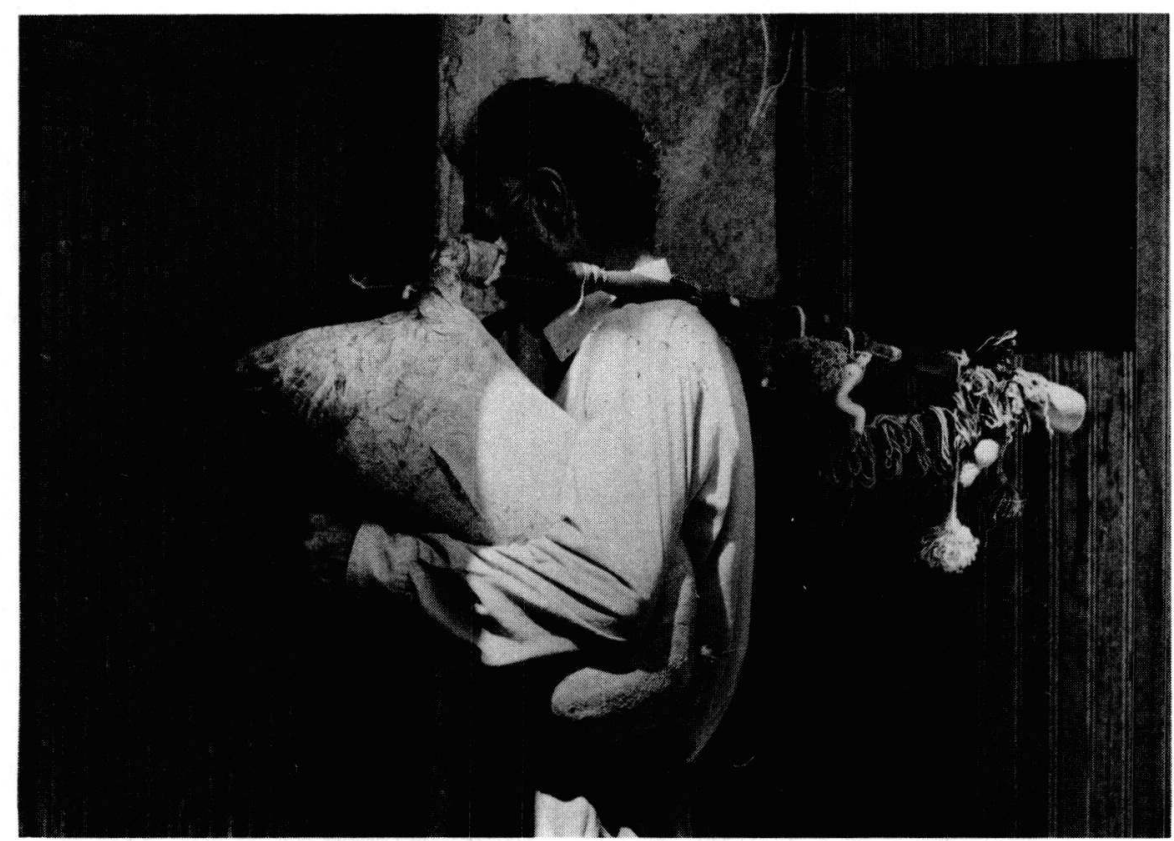

Fig. 4: A. Preto, cornemuseur du village de Caravela. Photo: A. Caufriez, 1983. 
huit trous mélodiques dont un, postérieur, est destiné au pouce, ainsi que trois trous d'accord ou de clarté. Il est joué avec trois doigts de la main gauche (index, majeur et annulaire) et quatre doigts de la main droite (index, majeur, annulaire et auriculaire).

Le chalumeau peut présenter de petites variantes selon les villages. Ainsi, la cornemuse transmontaine des collections du Musée instrumental de Bruxelles $^{3}$ ne possède que sept trous et un trou d'accord.

- Le bourdon (roncão) est un tuyau cylindrique composé de trois pièces (lanços) reliées les unes aux autres par le tenon s'encastrant dans la souche. La première partie (ombreira) est celle qui se place sous l'épaule du musicien et qui prend naissance dans la souche du réservoir d'air. L'extrémité inférieure de cette partie se termine par le tenon dans lequel est inséré le petit tuyau de roseau de l'anche. Celle-ci est simple, se caractérisant par une languette battante découpée dans le fût du roseau et dont la base est ligaturée avec du fil. La deuxième partie (intermeia ou bordão) s'encastre dans la précédente par le tenon. La troisième partie (copa, boca ou terceira) s'encastre dans la précédente par le tenon pour prendre la forme d'une cloche renversée à son extrémité supérieure.

Le réservoir d'air se place sous le bras gauche du musicien qui le serre avec le coude afin d'expulser l'air insufflé par le tuyau (assoprete). Cet air est alors mis en vibration par les anches du chalumeau (ponteiro) et du bourdon roncao. Ce dernier est toujours placé sur l'épaule gauche du musicien et dirigé vers l'arrière $e^{4}$.

\section{Les échelles mélodiques}

Autrefois, les échelles mélodiques de la cornemuse variaient d'un village à l'autre du Trás-os-Montes, chaque localité ayant sa façon de concevoir le chalumeau. Aujourd'hui, devant le nombre restreint d'instruments encore en usage, il est très difficile de dresser le tableau des échelles transmontaines.

Aucune cornemuse ne résonne de manière aussi particulière que celles de la région de Mirando do Douro, nous dit Podnos. Il nous donne les intervalles en cents d'une échelle de la région: celle de l'instrument de Cercio en 1936 (Podnos 1974: 63, 66).

3 Il s'agit d'une cornemuse de la région de Mirando do Douro, dont le réservoir est en peau de chèvre $\left(\mathrm{N}^{\circ}\right.$ de la collection: H.B. 118).

4 Nous avons réalisé des enquêtes et des enregistrements de musique dans les villages du haut Trás-os-Montes (1978, 1980, 1983), régions de Miranda do Douro, Bragance et Vinhais. Les cornemuseurs des plateaux mirandais, enregistrés en 1978, s'appellent P. Perreira João (66 ans), A. Garrido Brazo (66 ans) et J.F. Rodriguez (47 ans). Ceux des plateaux de Bragance, enregistrés en 1983, s'appellent A. Gil do Cubo (65 ans), A.A. Rodriguez (63 ans) et E.F. Veiga (42 ans). La description de l'instrument est basée sur l'observation de leurs cornemuses ainsi que celle du Musée Instrumentale. Seule la cornemuse de A.A. Rodriguez était une tumbal. 


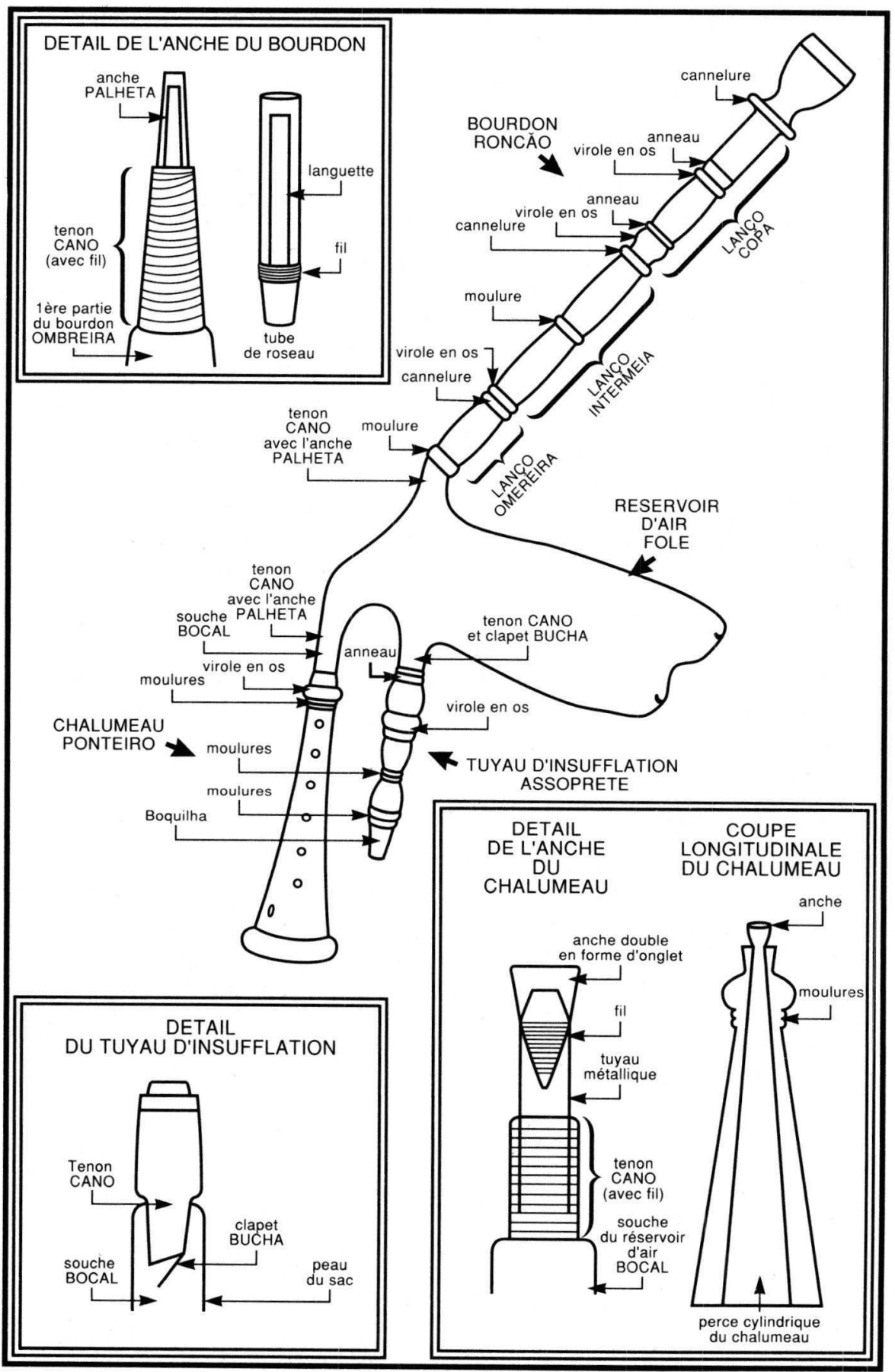

Dessin de la cornemuse $\mathrm{N}^{\circ} \mathrm{HB} 118$, du Musée Instrumental de Bruxelles. Dessin: Soheil Azzam. 
a \#'<smiles>C1CC2CCC(C1)C2</smiles>

125 $b^{\prime}$<smiles>C[13C](C)=[18O]</smiles>

c\#'

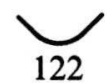

d'

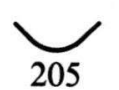

e'

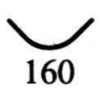

f \#

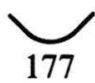

g'

En effet, les cornemuses des hauts plateaux mirandais produisent des échelles qu'on pourrait qualifier de non tempérées ou microchromatiques. Celles qu'on connaît le mieux, et qui remontent à une vingtaine d'années, sont dans les tons majeurs et mineurs anciens, présentant des diapasons variés.

Ces échelles caractéristiques de la région de Mirando do Douro et en partie de celle de Bragance ont cependant cédé la place à des échelles plus proches du tempérament égal. Citons, par exemple, celle du village de Moïmenta (région de Vinhais), dont les quartes et les quintes sont parfaites, la tierce légèrement fausse et la sixte neutre (Podnos 1974: 66).

Voici les échelles des cornemuses de Mirando do Douro (villages de Travanca et d'Ifanes) et de Bragance (village de Rio de Onor), telles que Veiga de Oliveira nous les rapporte dans les années 1960 (Oliveira 1966: 175):

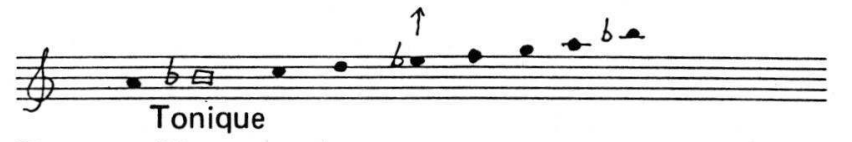

Travanca (Mogadouro) : mode de do avec quarte harmonique

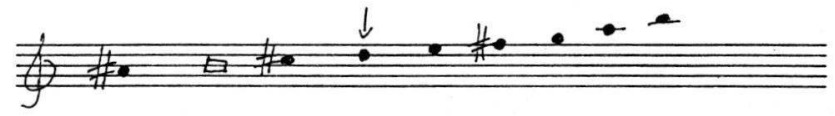

Ifanes (Miranda do Douro) : mode de la

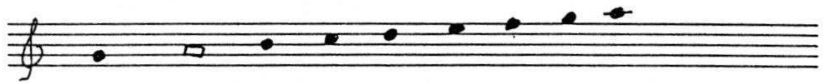

Rio de Onor (Bragance) : mode de la

Cette tradition d'échelles anciennes semble encore être partiellement vivante aujourd'hui, si l'on en juge la cornemuse du village de Póvoa (région de Mirando do Douro) que nous avons enregistrée. Elle produit l'échelle mineure que voici ${ }^{5}$ :

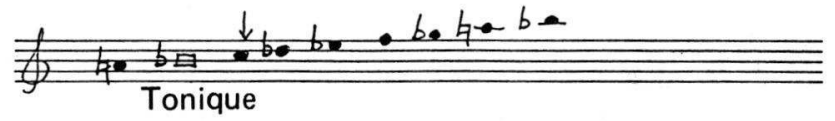

Les échelles mélodiques des cornemuses transmontaines sont donc très mobiles et flexibles, laissant apparaître des oscillations par rapport au modèle de base. Elles se rattachent plutôt au système modal que tonal, et elles ont leur exacte équivalence dans la musique vocale de la région ${ }^{6}$.

5 D'après H. Boone du Musée Instrumental de Bruxelles, les échelles mineures seraient très peu courantes dans les cornemuses actuelles d'Europe occidentale.

6 On rencontre des échelles tout à fait semblables dans les ballades que nous avons enregistrées au Trás-os-Montes en 1978, 1980 et 1983. 
Certains cornemuseurs du Trás-os-Montes enfoncent plus ou moins le tuyau de l'anche dans le tenon du chalumeau afin d'en modifier la longueur. Le changement d'emplacement de l'anche, tout comme d'ailleurs la variation de la pression sur le sac d'air peuvent, en effet, influencer la hauteur de certaines notes de l'échelle mélodique (Oliveira 1966: 176).

Dans la mesure où la cornemuse ancestrale existe encore, on peut dire qu'elle se distingue notamment par des échelles non tempérées et un ambitus restreint. C'est ce qui la rend plus archaïque que les cornemuses rencontrées actuellement en Galice espagnole, qui sont très nettement diatoniques. En outre, le style de la cornemuse transmontaine se caractérise par l'utilisation de trémolos et d'ornements qui amenuisent la stridence du timbre.

A la variété des échelles traditionnelles s'allie la variété des chalumeaux qui ne dépassent guère neuf notes, embrassant une octave à laquelle s'ajoute la sensible de l'octave inférieure grave.

Quant au diapason de l'instrument, celui-ci dépend notamment de la longueur et de la largeur du chalumeau, de l'évasement de la perce (une perce très évasée donne un diapason aigu) et de l'emplacement de l'anche dans celui-ci. Pour favoriser une belle sonorité, l'anche de la cornemuse requiert un milieu humide. C'est pourquoi, avant de jouer, beaucoup de musiciens transmontains laissent tremper les anches dans de l'eau de vie, leur bain préféré, ou dans l'eau salée ${ }^{7}$. Enfin, le bourdon s'accorde deux octaves plus bas que la tonique du chalumeau.

\section{L'accompagnement des tambours}

D'après nos informateurs, la cornemuse est toujours accompagnée du jeu des tambours bombo et caixa, mais elle est jouée seule par le berger qui garde son troupeau ou qui accompagne le chant des femmes lors des soirées de filage (Caufriez 1980: face A, plage 3).

Le bombo est un tambour en bois à deux membranes. Les peaux sont tendues sur une caisse cylindrique et reliées par un système de tension de cordes. Lorsque le fût de l'instrument est métallique, ce qui est de plus en plus fréquent, la tension des membranes est assurée par un système de tendons et de vis. Ce tambour est joué à l'aide d'une mailloche, et comme sa membrane vibre librement, sans corde chanterelle, il se distingue par une sonorité profonde et diffuse.

Quant à la caixa, il s'agit d'un tambour qui s'apparente au bombo, bien que de dimension plus réduite, avec une caisse de résonance plus épaisse. Sa technique de jeu l'en différencie: il se tient horizontalement (contrairement au bombo) et il est frappé à l'aide de deux baguettes en alternance. La caixa est aussi munie sur la peau inférieure, d'une ou de plusieurs cordes chanterelles s'apparentant à un bourdon. Celui-ci, généralement en boyau, est fixé à un régulateur qui en gradue la tension (Oliveira 1966: 188, 190).

7 Explications du cornemuseur de Caravela et de celui de Duas Igrejas. 


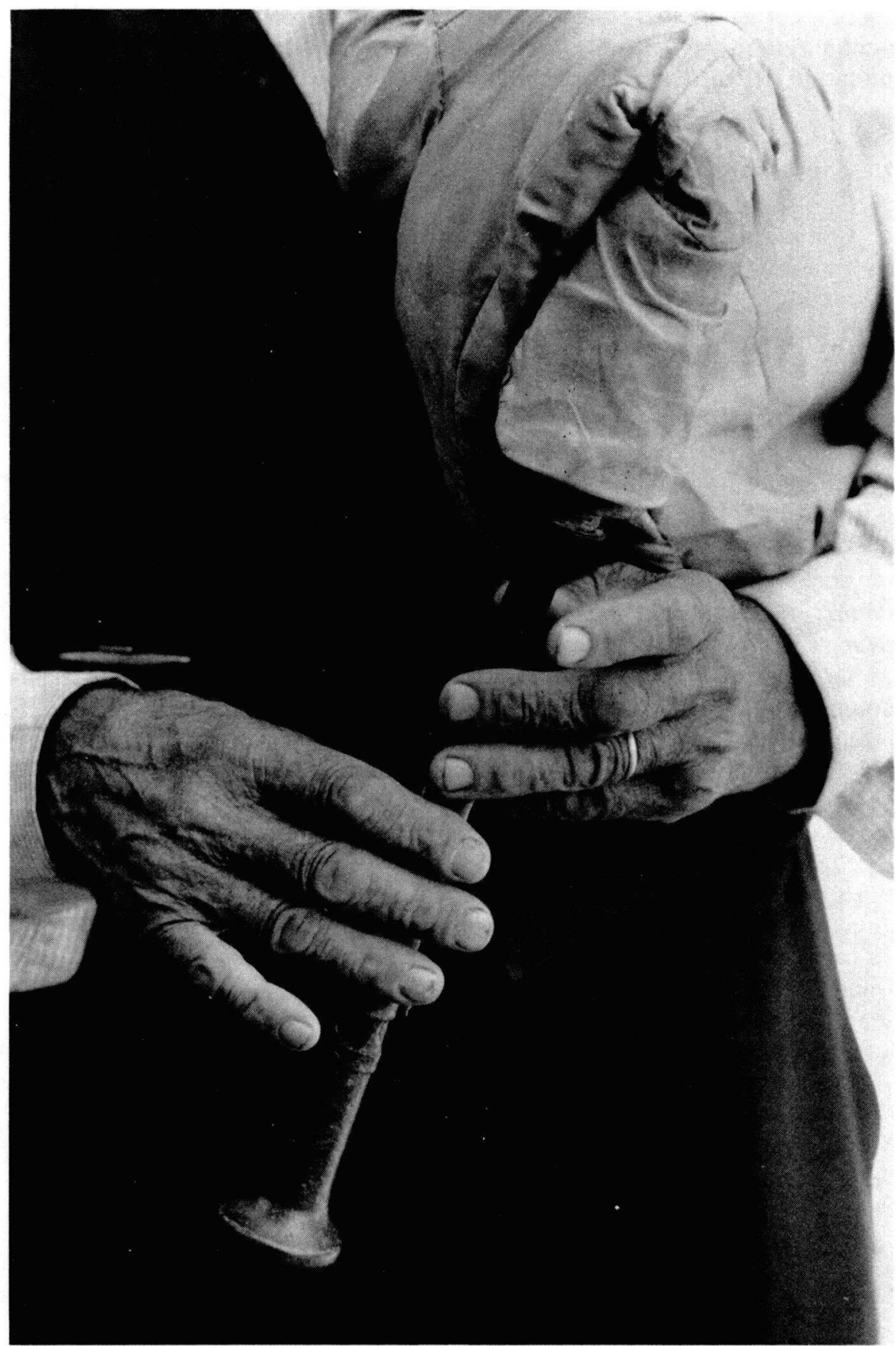

Fig. 5: A. Gil do Cubo, montrant la position des doigts sur le chalumeau. Photo: A. Caufriez, 1983. 
Le cornemuseur était occasionnellement accompagné du flûtistetambourinaire, tamborileiro. Le timbre cristallin des triangles (ferrinhos) et la pulsation des castagnettes (castanholas) s'y ajoutaient parfois.

\section{Les occasions du jeu}

Comme nous l'avons déjà dit, la cornemuse est l'attribut des bergers, Si elle est d'abord l'inséparable compagne des longues veillées de troupeaux, comme instrument de la collectivité villageoise elle est essentiellement investie de fonctions festives. Seul le chœur de l'église lui confère une sacralité.

\section{La liturgie et les fêtes du calendrier religieux}

Jusqu'à tout récemment, le cornemuseur avait son rôle dans la liturgie de la messe, où il jouait la mélodie des Santos, tandis que le prêtre donnait l'hostie.

L'air A Pascoa était joué, comme son nom l'indique, pour l'office de Pâques, tandis que Cremos Deus servait les processions se déroulant à l'extérieur de l'église.

La coutume d'associer le cornemuseur à la liturgie est aujourd'hui abandonnée sous la pression de l'ordre ecclésiastique, nous a affirmé le curé du village de Babe (Lombadas). Mais les mélodies liées à ces circonstances restent gravées dans la mémoire des musiciens, puisque nous en avons enregistré quelques-unes, ainsi que bon nombre d'airs de processions religieuses.

La cornemuse de la région mirandaise est surtout associée à la danse des pauliteiros, se manifestant aujourd'hui dans les zones lisières du nord-est du Portugal, mais aussi dans les provinces voisines de l'Espagne ${ }^{8}$. Les interprètes de cette danse sont exclusivement masculins. Ils servaient jadis les temps forts du calendrier religieux, comme les fêtes du Saint-Sacrement, celles de NotreDame du Rosaire, de Sainte-Barbe, de Saint-Sylvestre. Avant que l'angélus ne sonne, les danseurs exécutaient les figures appropriées à la circonstance devant les portres des maisons, tout en se livrant à la quête.

Certains pensent que la danse des páuliteiros est une ancienne danse guerrière qui, avec ses éléments de caractère militaire, est peut-être antérieure au Moyen Age (Mourinho 1957: 158, 164). D'autres y voient le reliquat d'une danse de fertilité. Toujours est-il qu'elle remplissait déjà une fonction importante dans les fêtes religieuses de la région au XIX ${ }^{\mathrm{e}}$ siècle (Baçal 1934: 511).

8 Paulitos signifie «bâtons» ou «bâtonnets». Pauliteiros signifie littéralement «joueurs de bâtons». En Espagne, on rencontrait cette danse en Galice, dans la région de Zamora, à Salamanque, à Léon (ville) et à Valladolid. Cette information remonte à 1949. Au Portugal, on ne rencontre la danse en question que dans le Trás-os-Montes (conseils de Miranda do Douro, Vimioso et Modagouro). 
Les figures lhaços de cette chorégraphie se singularisent par le jeu des bâtons paulitos percutés les uns contre les autres. Les danseurs, revêtus d'une jupe et de chaussettes rayées multicolores, en tiennent un dans chaque main (Oliveira 1966: 74, 75). Alors que l'appel du bombo est destiné à rassembler les danseurs (Giacometti 1964: face II, plage 5), chaque lhaço a ses paroles que les chorégraphes chantent, tout en exécutant les figures qui leur sont appropriées (Alves 1980: 148, 189). La danse est toujours accompagnée de la triade cornemuse-tambours (gaita de foles, bombo et caixa), auxquels sont adjoints les triangles (ferrinhos) et les castagnettes (castanholas) ${ }^{9}$. Bien que le groupe animé et entretenu par le Padre Mourinho continue à pratiquer cette danse, elle n'en était pas moins en déclin dans toute la région mirandaise vers 1980 , comme en témoigne le village de São Martinho d'Angeira. Jusqu'à récemment, en effet, il était très réputé pour son groupe de pauliteiros. Mais depuis que le cornemuseur a émigré en France, le groupe s'est dissocié sans pouvoir retrouver la qualité de ses prestations ${ }^{10}$. Les cornemuseurs que nous avons rencontrés connaissaient tous les airs de cette danse très formelle des Pauliteiros qui a imprégné la région, tels que: $O$ Mirandum, Lherba, Vinte e Cinco Aberto, $A$ Procissão et Acta da Contrição. Cette danse à caractère rituel ne subsiste-t-elle aujourd'hui que dans le jeu seul d'une seul cornemuse livrée à elle-même? (cf. Caufriez 1980: face B, plage 7).

Au Trás-os-Montes, le cornemuseur est aussi la figure centrale des festivités de Noël. A cette occasion, il joue un rôle actif dans la crèche vivante représentée dans le chœur de l'église. Cette tradition nous ramène à l'interprétation populaire des écritures, telle qu'on la rencontre à l'époque médiévale chez les Bénédictins, par exemple. On la connaît notamment par les peintres de la première renaissance italienne (fin du $X^{\text {e }}$ siècle).

Plus d'un village actuel reste attaché à l'intervention du cornemuseur pendant le cycle des douze jours. Et lorsque la localité peut encore se prévaloir de cet instrumentiste, les festivités de Noël continuent de représenter le cadre d'expression privilégié de celui-ci. Au sortir de la messe de Noël, la nuit est alors auréolée par les sortilèges d'une cornemuse qui se livre aussi bien aux airs des vieilles danses galiciennes, telle la murinheira, qu'aux jotas ou aux fandangos (cf. respectivement Caufriez 1980: face B: plages 5, 7, 1) ${ }^{11}$.

9 A noter que le mot lhaço, toujours utilisé par les cornemuseurs du Trás-os-Montes, vient du castillan. En portugais, lhaço devient laço. Les ferrinhos sont de petits triangles en fer, évidés, que le musicien percute avec une baguette (également en fer); ils introduisent un joli timbre dans la partie rythmique.

10 Nos enregistrements du groupe de ce village révèlent un état de décomposition avancé des répertoires de cette danse.

11 Rappelons que la danse de la murinheira était très populaire dans le Portugal des XIIIe et XIVe siècles. A murinheira est la désignation portugaise du Trás-os-Montes et signifie «meunière». En portugais courant et actuel, on dit plutôt a moleira (le moulin se dit o moinho). Le terme de murinheira remonte directement au mot galicien muhineira, lui-même dérivé de muhino («moulin»). En castillan, muhino devient molino, et muhineira, molineira. 
Au cycle des douze jours appartient aussi la Fête des garçons, rite de passage propre au Trás-os-Montes, et qui coïncide dans certains villages avec Noël ou l'Epiphanie. La cornemuse ne marque pas une classe d'âge unique, car elle seconde aussi la danse des vieux, en déclin (Oliveira 1966: 74). La fête des garçons est toujours vivante dans certains villages, et nous avons enregistré des répertoires qui lui sont propres, comme $A$ Marcha et $A$ Ronda.

Les sonorités stridentes de la cornemuse se font entendre encore pour le repas de plein air de la Saint-Etienne, alors que son souffle est inépuisable pour les banquets pantagruéliques et le bal de la Saint-Jean. Cette dernière fête coïncidant, comme on le sait, avec le solstice d'été, elle marque aussi dans cette région le début des moissons.

Enfin, toute fête est annoncée au village par l'air de l'alvorada (l'aube), que l'on joue dès l'aube aux portes des maisons. Ce répertoire commun avec la Galice est resté gravé dans la mémoire de tous les cornemuseurs de la région (Caufriez 1980: face A, plage 1).

\section{Les réjouissances collectives}

En dehors de ses fonctions cérémonielles, la cornemuse anime occasionnellement certains travaux agricoles ou artisanaux qui s'exécutent le soir devant l'âtre. Nous pensons au filage du lin ou au décorticage des châtaignes. Mais ces activités sont plutôt accompagnées du chant. Et nous arrivons aux réjouissances paysannes qui se déploient dans la vie profane.

Les occasions de bals champêtres sont nombreuses, allant du divertissement quotidien et dominical aux rites de passage et de fertilité. Comme nous l'avons déjà dit pour le cycle des douzes jours, la cornemuse assure la disjonction des classes d'âge mais elle suit aussi tout le déroulement des noces. Elle accompagne alors le banquet de famille qui se prolonge dans un bal nocturne.

D'autre part, la cornemuse ouvre et ferme véritablement le cycle des moissons de la région.

En effet, la fête de la Saint-Jean, coïncidant souvent avec le solstice d'été, marque le début de la période de fauchage. Les mélodies enjouées du berger s'éteignent alors avec les derniers feux pour céder la place aux chants de moisson. Mais l'instrument rejaillit parfois pour prolonger l'incandescence d'une journée de labeur dans un bal de crépuscule. La fin du cycle des moissons ainsi que celle du battage du grain est alors couronnée de grandes festivités au cœur desquelles l'instrument rend une ode à la nature (16).

Danses des batteurs de grain, des sarcleurs ou des moissonneurs, danses des filandières ou des amoureux du dimanche, danses des banquets de famille ou des foires régionales ... la cornemuse ne peut faillir à ses fonctions récréatives. Elle est avant tout un instrument de plein air.

La cornemuse enjouée du bal scande tout une série de danses allant des mélodies hiératiques du pingacho et du galandum à celles, plus fleuries, de la Galice médiévale, comme la murinheira, la carvalhesa, le passeado et le 
redondo. Bon nombre de fandangos et de jotas, s'associant aux mêmes circonstances, représentent les danses à succès venues d'Espagne ${ }^{12}$.

Danses ancestrales et danses modernes se côtoient et se mélangent dans toutes les fêtes villageoises. Hormis celles qui sont assignées à certains rituels religieux, elles se retrouvent indifféremment dans l'une ou l'autre circonstance. Le formalisme du choix ne saurait trouver sa place dans la ferveur collective du bal qui a ses temps forts et ses instants de respiration. La danse de la murinheira illustre bien cette perméabilité de la coutume, puisqu'elle était exécutée aussi bien pour la nuit de Noël que pour le divertissement dominical.

Les danses galiciennes appartiennent à un vieux fonds chorégraphique, si l'on se rappelle qu'elles servaient, au Moyen Age, à accueillir les rois et les nobles ou à désamorcer les guerres. La murinheira, par exemple, est connue dès le XIII' ${ }^{\mathrm{e}}$ siècle (Martins 1939: XLIII, vol. II; Riego 1963: 4, 5, 133, 136). La danse du pingacho, accompagnée d'un chœur, est en liaison avec d'archaïques rites de fertilité. Mais sa forme actuelle ne remonterait pas au-delà du XVII ${ }^{\mathrm{e}}$ ou XVIII ${ }^{\mathrm{e}}$ siècle (Mourinho, Bessa Bento \& Santos 1957: 1, 3, 7, 8). Quant au galandum, il aurait été importé d'Espagne par les moissonneurs et se rattacherait aux danses aristocratiques des $\mathrm{XVII}^{\mathrm{e}}$ et $\mathrm{XVIII}^{\mathrm{e}}$ siècles. La langue hybride de son chant trahit bien ses origines (Valentim, Mourinho \& Santos 1953: 1-3, $5,16)$. La carvalhesa, enfin, très difficile à danser, n'est plus l'apanage que de quelques vieux montagnards, alors que les figures de la murinheira sont un peu mieux conservées. Sur les six cornemuseurs de talent recensés lors de nos enquêtes, seuls deux ont encore pu jouer les mélodies des vieilles danses galiciennes, alors que tous avaient oublié le pingacho et le galandum ${ }^{13}$.

En revanche, une grande quantité de danses, plus récentes celles-là, font partie du répertoire des bergers, telles que: $O$ Loureiro, Zé Canedo, Helena, $O$ que lindo chapeu preto, Manolo Mio, Tia Ana, As Pombas da Catarina, O Diolinda, O Antonio, A Saia da Carolina. Ces danses peuvent parfois se rattacher au genre de la jota ou du fandango, bien que ces derniers soient plutôt présentées par les musiciens comme des répertoires autonomes. Voici la transcription musicale d'un fandango interprété par Paulino Perreira João, que nous avons enregistré au village de Póvoa en 1978:

12 La murinheira et la carvalhesa ont été enregistrées notamment au village de Paradinha (1978) et interprétées par Augusto Garrido Brazo. Le passeado a été enregistré au village de Pòvoa (1978) et interprété par Paolino Pereira João, cornemuseur, et par Paolino Raposo Oliveira et Alfredo Falcão, tambourinaires.

Cette observation se réfère à nos prospections de 1978 et 1983. 

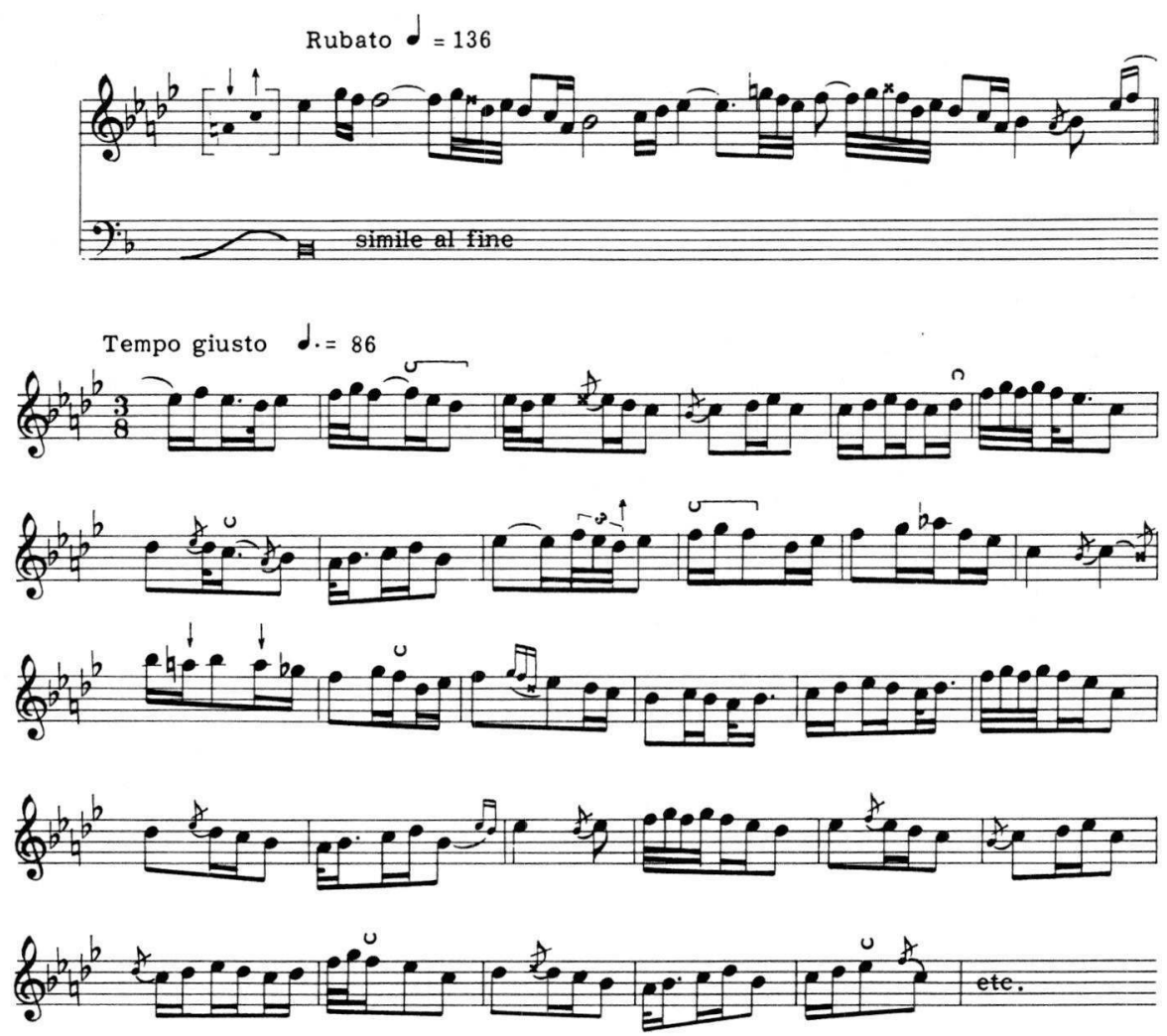

\section{En conclusion}

Si la cornemuse galicienne refleurit à travers des répertoires métamorphosés, celle du Trás-os-Montes n'a pas la même histoire. Les cornemuseurs transmontains, ces dépositaires de mélodies et de techniques de jeu parmi les plus anciennes de la Péninsule, se réduisent aujourd'hui à une douzaine de bergers pour l'ensemble des montagnes qui avaient si longtemps protégé leur art.

Des répertoires liturgiques ou de la danse des pauliteiros, si intime à l'identité mirandaise, il ne reste que l'écume des fêtes avec ces alvoradas (aubades) dont tous se souviennent. Alors que le musicien est toujours accueilli pour les festivités de Noël, le cycle des douze jours menace son existence à cause des villages désertés par les jeunes. Sa présence au bal de la Saint-Jean ou aux mariages est peu à peu supplantée par la musique amplifiée d'un cassettophone. Par ailleurs, l'émigration a aussi éparpillé les ensembles cornemuse-tambours, et peu de villages se réveillent encore au roulement des grosses caisses.

Les vieilles danses galiciennes ont perdu leurs acteurs, et plusieurs fêtes se privent maintenant de la cornemuse, tandis que le pâtre ne cherche plus à 
rompre le silence inquiétant des campagnes. Si la vieille cornemuse celtique abandonne peu à peu un village en crise de relais, elle est aussi attachée à une communauté paysanne dont la lutte pour la survie est devenue trop dure.

Pour le mélomane, certains espoirs sont toutefois permis, car des cornemuseurs peuvent encore sortir de l'anonymat, alors que d'autres reprennent leur instrument abandonné depuis dix ans. C'est l'histoire du musicien de Póvoa, qui après la fin de l'empire colonial, a dû quitter l'Angola pour retourner dans son Trás-os-Montes natal. Il avait perdu la maîtrise de sa cornemuse et se sentait mal intégré au village. Après notre visite de 1978, il reprit l'exercice de son instrument avec un engouement provoqué par une identité retrouvée. Aujourd'hui, il se déplace régulièrement au Portugal et surtout en Espagne pour animer des festivals. Et il semble que d'autres cornemuseurs de la région suivent ses traces.

\section{Bibliographie}

ALVES A.C.

1980 Em volta de uma espada. Glorias Mirandesas 1930. Miranda do Douro: Camara Municipal.

BAÇAL Abade de

1934 «Memorias arqueológico-históricas do Distrito de Bragança» G. da Assunção éd. Bragança, vol. IX, p. 511.

BAINES A.C.

1960 Bagpipes. Ed. par Pitt Rivers Museum. Oxford: Oxford University Press.

MARTINS A.F.

1939 Folclore do Conselho de Vinhais. Lisboa: Imprensa Nacional, Vol. II.

MOURINHO A.M. Padre

1957 «A dança dos paulitos». Revista Ocidente (Lisboa) LIII.

MOURINHO A.M. Padre, BESSA Bento \& SANTOS Junior

1957 "Coreografia popular Transmontana IV. O Pingacho». In: Douro Litoral. Boletim da Comissão de Etnografia e Historia (Porto), Oitava Série I-II.

OLIVEIRA E. Veiga de

1966 Instrumentos musicais populares portugueses. Lisboa: Fundação Calouste Gulbenkian. PODNOS T.H.

1974 Bagpipes and tunings. Detroit: Library of Congress Catalog.

RIEGO F. Fernandez del

1960 "A musica popular galega». In: Separata das Actas do Coloquio de Estudos Etnograficas, Dr J. Leite de Vasconcelos (Porto), vol. III.

1963 "As dansas populares galegas». In: Actas do $1^{\circ}$ Congresso de Etnografia e Folclore (Lisboa).

VALENTIM A. \& MOURINHO A.M. Padre

1953 "Coreografia popular transmontana III: O galandum». In: Douro Litoral (Porto), Quinto Série, VII-VIII. 


\section{Discographie}

CAUFRIEZ Anne

1980 Chants $d u$ blé et cornemuses de berger (Trás-os-Montes, Portugal). Paris: OCORA 558-547.

GIACOMETTI M.

1964 Visages du Portugal - Ethnologie vivante. Paris: Le Chant du Monde, LDX 4337. 